\title{
Nursing care for patients in post-transplantation of hematopoietic stem cells: an integrative review
}

\author{
Cuidados de enfermagem aos pacientes em pós-transplante de células-tronco hematopoiéticas: revisão integrativa
}

Atención de enfermería para pacientes en post-transplantación de células de madre hematopoyéticas: revisión integrativa

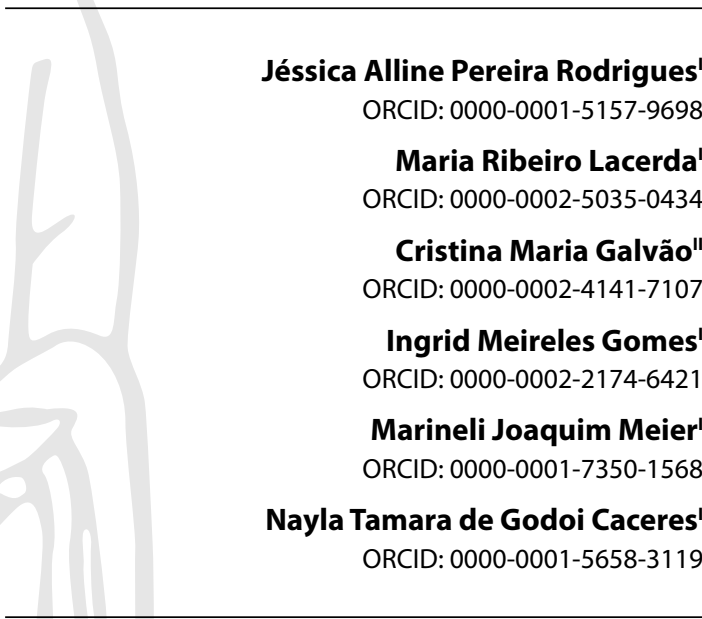

'Universidade Federal do Paraná. Curitiba, Paraná, Brazil. "Universidade de São Paulo. Ribeirão Preto, São Paulo, Brazil.

How to cite this article: Rodrigues JAP, Lacerda MR, Galvão MC, Gomes IM, Meier MJ, Caceres NTG. Nursing care for patients in post-transplantation of hematopoietic stem cells: an integrative review. Rev Bras Enferm. 2021;74(3):e20200097. https://doi.org/10.1590/0034-7167-2020-0097

Corresponding author: Jéssica Alline Pereira Rodrigues E-mail: jessica.rodrigues@hc.ufpr.br

EDITOR IN CHIEF: Dulce Barbosa ASSOCIATE EDITOR: Marcia Magro

Submission: $02-11-2020$

Approval: 02-07-2021

\section{ABSTRACT}

Objectives: to analyze the available evidence on the nursing care provided to patients after hematopoietic stem cell transplantation. Methods: integrative review with the search for primary studies in four databases and a virtual health library. A broad search strategy was used, including research published in English, Brazilian Portuguese, or Spanish, between 2008 and 2018 , totaling a sample of 42 studies. Results: the studies were grouped into three categories: multiple nursing care $(n=19)$, first-line care $(n=18)$, and self-management of care $(n=5)$. Conclusions: nursing care is critical, comprising patients' physical, psychological and social aspects. It occurs in hospital and home contexts, mainly involving technical actions and health guidance. The evidence identified provide subsidies for decision-making; however, most studies are of the non-experimental type, indicating the need for conducting intervention research.

Descriptors: Hematopoietic Stem Cell Transplantation; Bone Marrow Transplantation; Nursing Care; Professional Practice; Patient Care Planning.

\section{RESUMO}

Objetivos: analisar as evidências disponíveis sobre os cuidados de enfermagem realizados ao paciente em pós-transplante de células-tronco hematopoiéticas. Métodos: revisão integrativa com a busca de estudos primários em quatro bases de dados e uma biblioteca virtual na área da saúde. Foi utilizada estratégia de busca ampla, incluindo-se pesquisas publicadas em inglês, português ou espanhol, entre 2008 e 2018, totalizando uma amostra de 42 estudos. Resultados: os estudos foram agrupados em três categorias: cuidados multíplices de enfermagem $(n=19)$, cuidados de primeira linha $(n=18)$ e autogerenciamento do cuidado $(n=5)$. Conclusões: $o$ cuidado de enfermagem é crítico, compreendendo os aspectos físico, psicológico e social do paciente. Ele ocorre em contextos hospitalares e domiciliares, principalmente envolvendo ações técnicas e de orientações em saúde. As evidências identificadas fornecem subsídios para a tomada de decisão, entretanto a maioria dos estudos é do tipo não experimental, indicando a necessidade de condução de pesquisas de intervenção.

Descritores: Transplante de Células-Tronco Hematopoéticas; Transplante de Medula Óssea; Cuidados de Enfermagem; Prática Profissional; Planejamento de Assistência ao Paciente.

\section{RESUMEN}

Objetivos: analizar la evidencia disponible sobre los cuidados de enfermería brindados al paciente en el postrasplante de células madre hematopoyéticas. Métodos: revisión integradora con búsqueda de estudios primarios en cuatro bases de datos y biblioteca virtual en salud. Se utilizó una amplia estrategia de búsqueda, incluyendo investigaciones publicadas en inglés, portugués o español, entre 2008 y 2018, totalizando una muestra de 42 estudios. Resultados: los estudios se agruparon en tres categorías: cuidados múltiples de enfermería $(n=19)$, cuidados de primera línea $(n=18)$ y cuidados autogestionados $(n=5)$. Conclusiones: el cuidado de enfermería es crítico, entendiendo los aspectos físicos, psicológicos y sociales del paciente. Ocurre en contextos hospitalarios y domiciliarios, involucrando principalmente acciones técnicas y de orientación sanitaria. Las evidencias identificadas brindan subsidios para la toma de decisiones, sin embargo, la mayoría de los estudios son del tipo no experimental, lo que indica la necesidad de realizar investigación de intervención.

Descriptores: Trasplante de Células Madre Hematopoyéticas; Trasplante de Médula Ósea; Atención de Enfermería; Práctica Profesional; Planificación de Atención al Paciente. 


\section{INTRODUCTION}

Hematopoietic stem cell transplantation (HSCT) presents itself as a curative possibility for malignant and non-malignant diseases that affect the functioning of the bone marrow. In Brazil, between January and September 2019, 2,575 transplants were reported, with the states of São Paulo and Paraná having the highest number, with 1,253 and 268 , respectively. These procedures were carried out by 73 teams. The amount mentioned represents about 500 more procedures than in the same period in 2018, and 1,000 more when compared to $2016^{(1)}$. Despite the progressive growth, it is a complex procedure, with a high risk of morbidity and mortality; thus, differentiated clinical developments are expected, impacting the quantity of nursing care $^{(2-3)}$ as well as their possible diversity.

The complexity of carrying out the procedure is related to the fragility of patients' health condition and the need for special care, related to dietary restrictions, care with personal and environmental hygiene, social isolation, among others, and the risk of potentially fatal complications, such as infection, respiratory failure, metabolic disorders and graft versus host disease (GVHD) ${ }^{(4)}$, making nursing care determinant for treatment.

It is worth noting that, in a hospital environment, prior to discharge, nursing care also focuses on providing guidance, preparing patients and caregivers to develop home care. Therefore, care is complex ${ }^{(5-6)}$, mainly involving the technical and guidance actions of the nursing team members in charge of assistance.

The Brazilian scientific production that addresses nursing care at HSCT, especially intervention studies, is reduced. However, it is noteworthy that nurses play a decisive role in ensuring quality care for patients and their families. Furthermore, it is known that the evidence impacts clinical practice ${ }^{(5)}$.

In view of this, there are divergences in care practices between the different HSCT centers, and it is relevant to identify which care is actually performed by nursing in this context, punctuated in literature. Such knowledge will allow to identify the existing gaps regarding the types of studies and thematic areas of need, allowing the dissemination of synthesis of knowledge to nursing professionals and providing subsidies for the development of clinical practice. Additionally, it is understood that the results may assist in the decision-making of nurses and corroborate the implementation of actions for prevention, early identification and treatment of complications post-transplantation.

\section{OBJECTIVES}

To analyze the available evidence on the nursing care provided to patients after hematopoietic stem cell transplantation.

\section{METHODS}

The integrative literature review was used for the synthesis of knowledge, five steps were taken in conducting the method: development of the research question, searching the literature of primary studies, assessment of primary studies, data analysis and presentation of the review ${ }^{(7)}$.

To develop the review question, PICOT (patient, intervention, comparison, outcomes and time) was used. The use of such a strategy allows to assist the reviewer in identifying the body of evidence of interest ${ }^{(8)}$. Thus, the question of the formulated review was: what is the nursing care performed for patients in post-HSCT? The first element ( $\mathrm{P}=$ patient) consists of patients undergoing HSCT (any age), the second ( $I=$ intervention), in nursing care and the last element ( $\mathrm{T}=$ time), post-transplant period. It is noteworthy that the elements $C$ (comparison between intervention or group) and $O$ (outcomes/results) were not used considering the type of review.

The search for primary studies took place in four databases, PubMed of National Library of Medicine (PubMed), Cumulative Index to Nursing \& Allied Health Literature (CINAHL), Web of Science and Scopus, and the Virtual Health Library Nursing (Nursing $\mathrm{VHL}$ ). The descriptors were established considering the terms of the different databases/library, and the Mesh Database, including the entry terms, to ensure a broad search, associated with the acronym PICOT designed to assist in the search strategy of primary studies, in order to increase the sensitivity of this strategy. To search for publications, AND and OR Boolean operators were used. Chart 1 presents the strategy used as a basis for the search for studies, which was adapted according to the database consulted, and for this stage of the study, it was assisted by a librarian.

Primary studies on nursing care performed for patients in post HSCT, published in Brazilian Portuguese, English, or Spanish, and from January 2008 to December 2018 were included. The time frame was established to avoid a high amount of studies. Such a situation could render an analysis of the generated evidence unfeasible and introduce bias in the conduct of the following review stages.

Chart 1 - Strategy used as a basis for the search for primary studies in the different databases/library, Curitiba, Paraná, Brazil, 2019

\begin{tabular}{|c|c|}
\hline $\begin{array}{l}\text { Database/ } \\
\text { Library }\end{array}$ & Search strategy \\
\hline PubMed & \multirow[b]{5}{*}{ 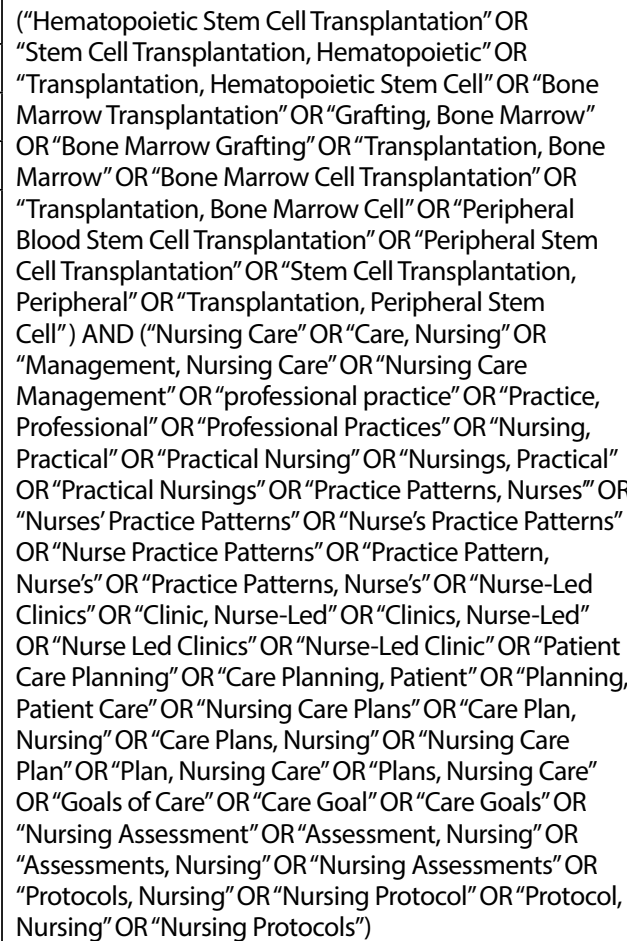 } \\
\hline Nursing VHL & \\
\hline $\mathrm{CINAHL}^{\dagger}$ & \\
\hline Web of Science & \\
\hline Scopus & \\
\hline
\end{tabular}
Health Literature. 
The results from the search strategy employed were imported into the reference manager EndNote Web. Folders were created for each database/library, with filtering for duplicate studies, books, dissertations, theses and other non-scientific texts and, with period of publication outside the established. Language filtering happened, manually, when reading the studies, to ensure greater accuracy in the selection, as well as new filtering referring to the duplicity of studies in situations in which the reference manager might not recognize.

Pre-selection of primary studies was carried out by reading the titles and abstracts, with subsequent selection for reading in full. Both steps were carried out by two researchers, independently, in order to reduce the selection bias of studies, with disagreements in the selection process being discussed. The publications in which the inclusion criteria were not clear when reading titles and abstracts were all read in full.

An instrument, developed by Brazilian researchers, was used to extract data from primary studies, which allows the registration of information on the identification of research, methodological characteristics, main results and conclusions ${ }^{(9)}$. A synthesis chart was prepared for each of the primary studies included in the review with a focus on nursing care.

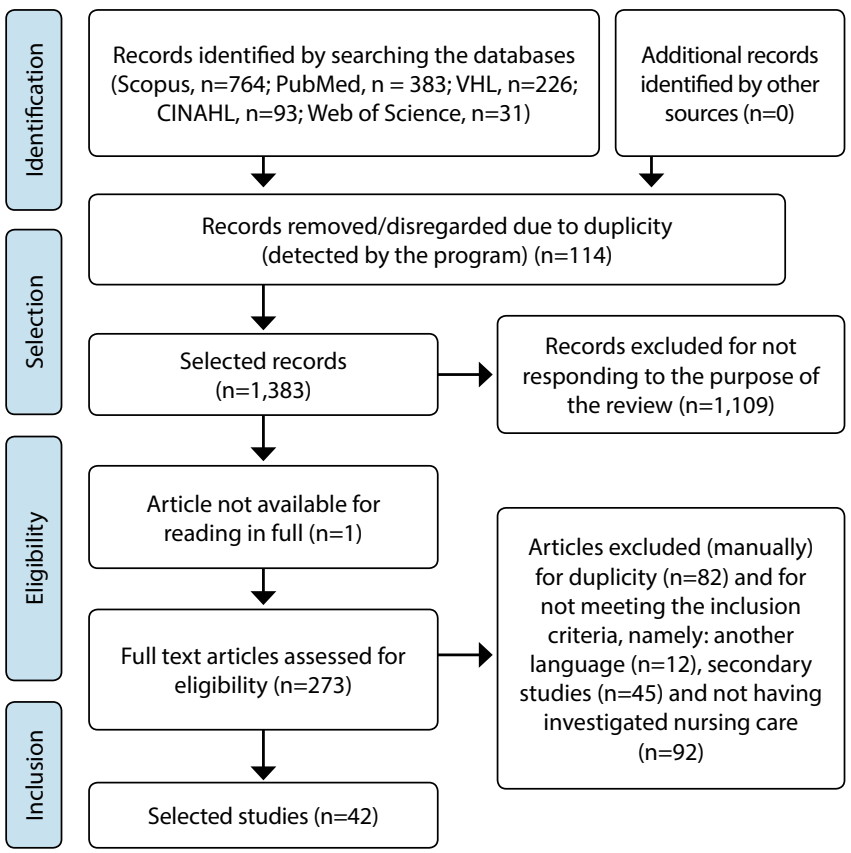

Figure 1 - Flowchart adapted from Preferred Reporting Items for Systematic Reviews and Meta-Analyzes on the description of the selection process of publications in the databases by combining descriptors and using filters, Curitiba, Paraná, Brazil, 2019

The indication of the methodological approach and the research design was based on the description of the studies' authors. In situations where the information was not described, the concepts of nursing researchers on scientific methodology were adopted ${ }^{(10)}$.

To aid in the assessment of primary studies, these were classified according to evidence hierarchies ${ }^{(8)}$ depending on the type of clinical question of the research, namely: (a) clinical question of meaning (with five levels of evidence, the strongest being , i.e., level l, evidence obtained from meta-synthesis of qualitative studies); (b) clinical question of prognosis, prediction or etiology (with five levels of evidence, the strongest being, i.e., level I, evidence obtained from the synthesis of cohort or casecontrol studies); (c) clinical question of intervention, treatment or diagnostic/diagnostic test (with seven levels of evidence, the strongest being, i.e., level I, evidence obtained from systematic review or meta-analysis of randomized clinical trials).

Figure 1 shows the study search and selection flowchart. The review sample consisted of 42 primary studies, which were grouped into three categories. Analysis of the review results was carried out descriptively.

\section{RESULTS}

Of the selected studies, for full assessment, access to a publication was not obtained, even for purchase, and there was no response to the request made to the authors. Therefore, the review sample consisted of 42 primary studies, eight of which were published in 2014 and seven in 2016 (years with the largest number of published studies). Regarding the country of origin, considering the main author, 17 studies are from the United States of America (USA), and 11 , from Brazil, with the other countries having the lowest number of publications. Regarding the journal's knowledge area, 28 studies were published in nursing journals, and 14 in medical ones.

In the review sample, 21 primary studies were classified as having a clinical question of prognosis/prediction or etiology, three with level of evidence II, and 18, level IV. In 15, the clinical question was intervention/treatment or diagnostic/diagnostic test, three with level of evidence II, 11 with level of evidence III, and one with level IV. In six studies, the clinical question was classified as meaningful, all with evidence level II.

As already mentioned, primary studies were grouped into three categories, which were named according to the characteristics of the investigated care, namely: 1$)$ multiple nursing care $(n=19)$, in this category, the research whose authors studied different care common to the HSCT routine were listed; 2 ) first-line care $(n=18)$, in this category were grouped studies whose researchers investigated fundamental care, which, when not performed, put patients'lives at risk, including preventive infection care $(n=8)$, care with central venous catheter (CVC) $(n=6)$ and care with mucositis $(n=4) ; 3)$ self-management of care $(n=5)$, in this category, studies on patient preparation to perform self-care were discussed.

Chart 2 presents the characterization of primary studies grouped in the multiple nursing care category $(n=19)$.

Chart 3 presents the characterization of primary studies grouped in the category first-line care $(n=18)$.

In the self-management of care category, five primary studies were grouped, being presented in Chart 4.

Nurses were nominally cited as responsible for the care listed in 26 of the primary studies $(2,13,15-17,19-21,23-24,27,29,32-39,41,44-45,48-50)$, and in the remaining $16^{(5,11-12,14,18,22,25-26,28,30-31,40,42-43,46-47)}$, the nursing team was mentioned.

Technical care (involving skills developed by professionals) occurred in 26 of the surveys included in the review $^{(2,11,13-14,17-18,20-22,27-39,41,45-46,50)}$. Care guidance, for execution by patients and family members at home, was investigated in five studies $^{(15-16,23,47-48)}$. The combination of technical care and guidance provided was thematic studied in 11 studies $^{(5,12,19,24-26,40,42-44,49)}$. 
Chart 2 - Characterization of primary studies in the category multiplicity nursing care $(n=19)$, according to author(s), year of publication and country, type of study, intervention/focus, clinical issue and level of evidence, Curitiba, Paraná, Brazil

\begin{tabular}{|c|c|c|c|c|}
\hline Author(s) & Year/country & Type of study & Intervention/focus & $\begin{array}{l}\text { Clinical question/ } \\
\text { level of evidence }\end{array}$ \\
\hline Agulnik et al. ${ }^{(11)}$ & $\begin{array}{l}2016 / \\
\text { United States } \\
\text { of America }\end{array}$ & $\begin{array}{l}\text { Case-control study, comparative } \\
N=110 \text { (transfers) } \\
N=220 \text { (control) }\end{array}$ & $\begin{array}{l}\text { Ability of the Pediatric Early Warning Score to predict } \\
\text { unplanned transfer of patients to the Pediatric } \\
\text { Intensive Care Unit. }\end{array}$ & $\begin{array}{l}\text { Intervention/ } \\
\text { treatment or diagnosis/ } \\
\text { diagnostic test//V* }\end{array}$ \\
\hline Araujo et al. ${ }^{(12)}$ & $\begin{array}{l}2015 / \\
\text { Brazil }\end{array}$ & $\begin{array}{l}\text { Descriptive-exploratory, } \\
\text { quantitative study } \\
\mathrm{n}=30\end{array}$ & $\begin{array}{l}\text { Main diagnoses and nursing interventions for } \\
\text { patients with graft-versus-host disease. }\end{array}$ & $\begin{array}{l}\text { Prognosis/ } \\
\text { prediction or etiology/IV† }\end{array}$ \\
\hline Bergkvist et al. ${ }^{(13)}$ & $\begin{array}{l}\text { 2013/ } \\
\text { Sweden }\end{array}$ & $\begin{array}{l}\text { Quasi-experimental study, } \\
\text { control group not equivalent } \\
n=41\end{array}$ & $\begin{array}{l}\text { Satisfaction and experiences of care and support to } \\
\text { patients during the acute phase after transplantation, } \\
\text { when treated in the hospital or at home. }\end{array}$ & $\begin{array}{l}\text { Intervention/ } \\
\text { treatment or diagnosis/ } \\
\text { diagnostic test/lll }\end{array}$ \\
\hline Cater et al. ${ }^{(14)}$ & $\begin{array}{l}\text { 2018/ } \\
\text { United States } \\
\text { of America }\end{array}$ & $\begin{array}{l}\text { Retrospective, single-center } \\
\text { cohort study } \\
\mathrm{N}=102\end{array}$ & $\begin{array}{l}\text { Validity of the Pediatric Early Warning Score in } \\
\text { identifying patients who require critical intervention. }\end{array}$ & $\begin{array}{l}\text { Prognosis/ } \\
\text { prediction or etiology/ll§ }\end{array}$ \\
\hline Cooke et al. ${ }^{(15)}$ & $\begin{array}{l}2012 / \\
\text { United States } \\
\text { of America }\end{array}$ & $\begin{array}{l}\text { Qualitative study } \\
\mathrm{N}=141\end{array}$ & $\begin{array}{l}\text { Discussion of topics (diet, physical symptoms, } \\
\text { emotional issues) that arose from spontaneous } \\
\text { concerns of patients after teaching intervention. }\end{array}$ & Meaning/II\| \\
\hline Cooke et al. ${ }^{(16)}$ & $\begin{array}{l}\text { 2011/ } \\
\text { United States } \\
\text { of America }\end{array}$ & $\begin{array}{l}\text { Quasi-experimental study } \\
\text { (no control group) } \\
\mathrm{N}=4\end{array}$ & $\begin{array}{l}\text { Application of the content principles of End-of-life } \\
\text { Nursing Education Consortium. }\end{array}$ & $\begin{array}{l}\text { Intervention/ } \\
\text { treatment or diagnosis/ } \\
\text { diagnostic test/Il| }\end{array}$ \\
\hline Ferreira et al. ${ }^{(17)}$ & $\begin{array}{l}2017 / \\
\text { Brazil }\end{array}$ & $\begin{array}{l}\text { Descriptive, qualitative study } \\
\mathrm{N}=9\end{array}$ & $\begin{array}{l}\text { Nurses' experiences on competencies necessary for } \\
\text { patient care. }\end{array}$ & Meaning/II\| \\
\hline Gori et al..$^{(18)}$ & $\begin{array}{l}\text { 2014/ } \\
\text { Italy }\end{array}$ & $\begin{array}{l}\text { Descriptive study } \\
\mathrm{N}=48 \text { centers }\end{array}$ & $\begin{array}{l}\text { Application of Evidence-Based Practice to improve } \\
\text { clinical practice. }\end{array}$ & $\begin{array}{l}\text { Prognosis/ } \\
\text { prediction or etiology/IV }+\end{array}$ \\
\hline Kirsch et al. ${ }^{(19)}$ & $\begin{array}{l}\text { 2014/ } \\
\text { Switzerland }\end{array}$ & $\begin{array}{l}\text { Descriptive study } \\
N=143\end{array}$ & $\begin{array}{l}\text { Practice patterns of assessment/screening methods } \\
\text { and interventions used to improve medication } \\
\text { adherence. }\end{array}$ & $\begin{array}{l}\text { Prognosis/ } \\
\text { prediction or etiology/IV }+\end{array}$ \\
\hline Lima et al. ${ }^{(20)}$ & $\begin{array}{l}\text { 2014/ } \\
\text { Brazil }\end{array}$ & $\begin{array}{l}\text { Descriptive, qualitative study } \\
\mathrm{N}=12\end{array}$ & Nurses' care activities. & Meaning/II\| \\
\hline Lu et al. ${ }^{(21)}$ & $\begin{array}{l}\text { 2016/ } \\
\text { United States } \\
\text { of America }\end{array}$ & $\begin{array}{l}\text { Randomized study with two } \\
\text { groups } \\
\mathrm{N}=13 \text { (intervention) } \\
\mathrm{N}=13 \text { (control) }\end{array}$ & $\begin{array}{l}\text { Feasibility of the use of therapies similar to relaxation } \\
\text { and relaxation therapy. }\end{array}$ & $\begin{array}{l}\text { Intervention/ } \\
\text { treatment or diagnosis/ } \\
\text { diagnostic test/II }\end{array}$ \\
\hline Meseguer-Liza et al. ${ }^{(22)}$ & $\begin{array}{l}\text { 2010/ } \\
\text { Spain }\end{array}$ & $\begin{array}{l}\text { Prospective descriptive study } \\
\mathrm{N}=10\end{array}$ & Reliability of the new fever diagnostic method. & $\begin{array}{l}\text { Prognosis/ } \\
\text { prediction or etiology/lV } \dagger\end{array}$ \\
\hline Montgomery et al..(23) & $\begin{array}{l}\text { 2016/ } \\
\text { United States } \\
\text { of America }\end{array}$ & $\begin{array}{l}\text { Cross-sectional descriptive study } \\
\mathrm{N}=130\end{array}$ & $\begin{array}{l}\text { Description of perceptions of health care providers in } \\
\text { relation to enteral and parenteral nutrition. }\end{array}$ & $\begin{array}{l}\text { Prognosis/ } \\
\text { prediction or etiology/IV }+\end{array}$ \\
\hline Rodrigues et al. ${ }^{(24)}$ & $\begin{array}{l}2013 / \\
\text { Portugal }\end{array}$ & $\begin{array}{l}\text { Exploratory-descriptive, } \\
\text { quantitative, retrospective study } \\
\mathrm{N}=122 \text { (hospitalizations) }\end{array}$ & $\begin{array}{l}\text { Profile of patients submitted to HSCT and } \\
\text { identification of the standard of care documented } \\
\text { by nurses. }\end{array}$ & $\begin{array}{l}\text { Prognosis/ } \\
\text { prediction or etiology/lV } \dagger\end{array}$ \\
\hline Rodrigues et al. ${ }^{(25)}$ & $\begin{array}{l}2018 / \\
\text { Brazil }\end{array}$ & $\begin{array}{l}\text { Quantitative, retrospective, } \\
\text { cross-sectional study } \\
\mathrm{N}=138\end{array}$ & Nursing care for children in post-HSCT. & $\begin{array}{l}\text { Prognosis/ } \\
\text { prediction or etiology/IV }+\end{array}$ \\
\hline Silva et al. ${ }^{(26)}$ & $\begin{array}{l}2015 / \\
\text { Brazil }\end{array}$ & $\begin{array}{l}\text { Prospective cohort study } \\
\mathrm{N}=62\end{array}$ & Nursing workload & $\begin{array}{l}\text { Prognosis/ } \\
\text { prediction or etiology/lI§ }\end{array}$ \\
\hline Valizadeh et al. ${ }^{(27)}$ & $\begin{array}{l}\text { 2014/ } \\
\text { Iran }\end{array}$ & $\begin{array}{l}\text { Qualitative study } \\
\mathrm{N}=18\end{array}$ & Strategies used by nurses to communicate bad news. & Meaning/II\| \\
\hline Vasquenza et al. ${ }^{(28)}$ & $\begin{array}{l}2015 / \\
\text { United States } \\
\text { of America }\end{array}$ & $\begin{array}{l}\text { Descriptive study } \\
\mathrm{N}=51\end{array}$ & $\begin{array}{l}\text { Pain control practices and use of patient-controlled } \\
\text { analgesia. }\end{array}$ & $\begin{array}{l}\text { Prognosis/ } \\
\text { prediction or etiology/IV }+\end{array}$ \\
\hline Zamanzadeh et al. ${ }^{(29)}$ & $\begin{array}{l}2013 / \\
\text { Iran }\end{array}$ & $\begin{array}{l}\text { Qualitative content analysis } \\
\mathrm{N}=18\end{array}$ & Emotional work experienced by nurses. & Meaning/II\| \\
\hline
\end{tabular}

Note: *evidence from cohort or case-control studies; † evidence of a single qualitative or descriptive study; $\neq$ fevidence of clinical trials without randomization; §evidence from a single cohort or casecontrol study; |levidence from a single qualitative study; fevidence from randomized clinical trials. 
Chart 3 - Characterization of primary studies of the first-line care category $(n=18)$, according to author(s), year of publication and country, type of study, intervention/focus, clinical issue and level of evidence, Curitiba, Paraná, Brazil

\begin{tabular}{|c|c|c|c|c|}
\hline Author (s) & $\begin{array}{l}\text { Year/ } \\
\text { country }\end{array}$ & Type of study & Intervention/focus & $\begin{array}{l}\text { Clinical question/ } \\
\text { level of evidence }\end{array}$ \\
\hline Balian et al. ${ }^{(30)}$ & $\begin{array}{l}2018 / \\
\text { United States } \\
\text { of America }\end{array}$ & $\begin{array}{l}\text { Retrospective cohort study } \\
\mathrm{N}=78\end{array}$ & $\begin{array}{l}\text { Incidence of bloodstream infection associated with } \\
\text { mucosal barrier injury and the relationship between } \\
\text { fecal organisms. }\end{array}$ & $\begin{array}{l}\text { Prognosis/ } \\
\text { prediction or etiology/ll* }\end{array}$ \\
\hline Barrell et al. ${ }^{(31)}$ & $\begin{array}{l}2012 / \\
\text { United States } \\
\text { of America }\end{array}$ & $\begin{array}{l}\text { Quasi-experimental study, } \\
\text { time series model } \\
\mathrm{N}=90\end{array}$ & Catheter-associated bloodstream infection rates. & $\begin{array}{l}\text { Intervention/ } \\
\text { treatment or diagnosis/ } \\
\text { diagnostic test/III }\end{array}$ \\
\hline Bevans et al. ${ }^{(5)}$ & $\begin{array}{l}\text { 2009/ } \\
\text { United States } \\
\text { of America }\end{array}$ & $\begin{array}{l}\text { Cross-sectional study } \\
\mathrm{N}=205\end{array}$ & $\begin{array}{l}\text { Variation in nursing practice with emphasis on } \\
\text { infection control. }\end{array}$ & $\begin{array}{l}\text { Prognosis/ } \\
\text { prediction or etiology/IV } \neq\end{array}$ \\
\hline Daniels et al..$^{(2)}$ & $\begin{array}{l}\text { 2018/ } \\
\text { United States } \\
\text { of America }\end{array}$ & $\begin{array}{l}\text { Quasi-experimental study, } \\
\text { time series model } \\
\mathrm{N}=8\end{array}$ & $\begin{array}{l}\text { Mean time of antibiotic administration in patients } \\
\text { admitted to the HSCT unit with initial fever. }\end{array}$ & $\begin{array}{l}\text { Intervention/ } \\
\text { treatment or diagnosis/ } \\
\text { diagnostic test/IIt }\end{array}$ \\
\hline Hou et al. ${ }^{(32)}$ & $\begin{array}{l}\text { 2013/ } \\
\text { China }\end{array}$ & $\begin{array}{l}\text { Retrospective study } \\
\mathrm{N}=44\end{array}$ & $\begin{array}{l}\text { Relationship between graft disease versus acute } \\
\text { host and intestinal infection. }\end{array}$ & $\begin{array}{l}\text { Prognosis/ } \\
\text { prediction or etiology/IV } \neq\end{array}$ \\
\hline Livadiotti et al..(33) & $\begin{array}{l}2012 / \\
\text { Italy }\end{array}$ & $\begin{array}{l}\text { Nonexperimental study } \\
\mathrm{N}=49 \text { (oncology units) }\end{array}$ & $\begin{array}{l}\text { Current practice of antimicrobial chemotherapy and } \\
\text { chemoprophylaxis for febrile neutropenia. }\end{array}$ & $\begin{array}{l}\text { Prognosis/ } \\
\text { prediction or etiology/IV } \neq\end{array}$ \\
\hline Shelton et al. ${ }^{(34)}$ & $\begin{array}{l}2016 / \\
\text { United States } \\
\text { of America }\end{array}$ & $\begin{array}{l}\text { Quasi-experimental study, } \\
\text { time series model } \\
\mathrm{N}=40 \text { (control) } \mathrm{N}=79 \\
\text { (intervention) }\end{array}$ & $\begin{array}{l}\text { Time between the initial presentation of patients } \\
\text { in the hospital, with possible sepsis (fever, } \\
\text { neutropenia) until the first intervention. }\end{array}$ & $\begin{array}{l}\text { Intervention/ } \\
\text { treatment or diagnosis/ } \\
\text { diagnostic test/IIt }\end{array}$ \\
\hline Vokurka et al..$^{(35)}$ & $\begin{array}{l}\text { 2014/ } \\
\text { Czech } \\
\text { republic }\end{array}$ & $\begin{array}{l}\text { Observational, prospective, } \\
\text { comparative, multicenter study } \\
\mathrm{N}=689\end{array}$ & $\begin{array}{l}\text { High efficiency particulate air filtration and its } \\
\text { impact on the incidence of pneumonia and } \\
\text { mortality. }\end{array}$ & $\begin{array}{l}\text { Prognosis/ } \\
\text { prediction or etiology/IV } \neq\end{array}$ \\
\hline Andrade et al. ${ }^{(36)}$ & $\begin{array}{l}2011 / \\
\text { Brazil }\end{array}$ & $\begin{array}{l}\text { Retrospective, exploratory, } \\
\text { descriptive study } \\
\mathrm{N}=68\end{array}$ & $\begin{array}{l}\text { Use and cost of different types of covers in central } \\
\text { venous catheter in relation to the frequency of } \\
\text { exchange and frequency of infections. }\end{array}$ & $\begin{array}{l}\text { Prognosis/ } \\
\text { prediction or etiology/IV } \neq\end{array}$ \\
\hline Silveira et al. ${ }^{(37)}$ & $\begin{array}{l}\text { 2010/ } \\
\text { Brazil }\end{array}$ & $\begin{array}{l}\text { Case series } \\
\mathrm{N}=10\end{array}$ & $\begin{array}{l}\text { Frequency of infection, related to catheter, and skin } \\
\text { toxicity, to the use of polyurethane dressing. }\end{array}$ & $\begin{array}{l}\text { Prognosis/ } \\
\text { prediction or etiology/lV } \neq\end{array}$ \\
\hline Flynn et al. ${ }^{(38)}$ & $\begin{array}{l}2015 / \\
\text { Australia }\end{array}$ & $\begin{array}{l}\text { Comparative study of two } \\
\text { groups without competing } \\
\text { controls } N=150\end{array}$ & $\begin{array}{l}\text { Assessment of change in practice on the connector } \\
\text { without needle in a central catheter associated with } \\
\text { the occurrence of infection. }\end{array}$ & $\begin{array}{l}\text { Intervention/ } \\
\text { treatment or diagnosis/ } \\
\text { diagnostic test/III } \dagger\end{array}$ \\
\hline Keeler ${ }^{(39)}$ & $\begin{array}{l}2014 / \\
\text { United States } \\
\text { of America }\end{array}$ & $\begin{array}{l}\text { Non-experimental study } \\
\mathrm{N}=25 \text { centers }\end{array}$ & $\begin{array}{l}\text { Adhering to the recommended guidelines for } \\
\text { central catheter management. }\end{array}$ & $\begin{array}{l}\text { Prognosis/ } \\
\text { prediction or etiology/IV } \neq\end{array}$ \\
\hline Carvalho et al..$^{(40)}$ & $\begin{array}{l}\text { 2014/ } \\
\text { Brazil }\end{array}$ & $\begin{array}{l}\text { Documentary research } \\
\mathrm{N}=3\end{array}$ & $\begin{array}{l}\text { Standardization of the central venous catheter } \\
\text { dressing technique. }\end{array}$ & $\begin{array}{l}\text { Prognosis/ } \\
\text { prediction or etiology/IV } \neq\end{array}$ \\
\hline Yilmaz et al. ${ }^{(41)}$ & $\begin{array}{l}\text { 2012/ } \\
\text { Turkey }\end{array}$ & $\begin{array}{l}\text { Prospective study } \mathrm{N}=114 \\
\text { (catheters) }\end{array}$ & $\begin{array}{l}\text { Types, rates and risk factors for complications related } \\
\text { to central catheters. }\end{array}$ & $\begin{array}{l}\text { Prognosis/ } \\
\text { prediction or etiology/IV } \neq\end{array}$ \\
\hline Bhatt et al. ${ }^{(42)}$ & $\begin{array}{l}2010 / \\
\text { United States } \\
\text { of America }\end{array}$ & $\begin{array}{l}\text { Quasi-experimental study, } \\
\text { time series model } \\
\mathrm{N}=13 \text { (control) } \\
\mathrm{N}=12 \text { (intervention) }\end{array}$ & $\begin{array}{l}\text { Implementation of a standardized oral care protocol } \\
\text { for the prevention and management of mucositis. }\end{array}$ & $\begin{array}{l}\text { Intervention/ } \\
\text { treatment or diagnosis/ } \\
\text { diagnostic test/IIt }\end{array}$ \\
\hline Braga et al. ${ }^{(43)}$ & $\begin{array}{l}\text { 2015/ } \\
\text { Brazil }\end{array}$ & $\begin{array}{l}\text { Randomized clinical trial } \\
\mathrm{N}=40\end{array}$ & $\begin{array}{l}\text { Chamomilla recutita dosage in mouthwash required } \\
\text { to reduce incidence and intensity of mucositis. }\end{array}$ & $\begin{array}{l}\text { Intervention/ } \\
\text { treatment or diagnosis/ } \\
\text { diagnostic test/II }\end{array}$ \\
\hline Leppla et al. ${ }^{(44)}$ & $\begin{array}{l}2016 / \\
\text { Switzerland }\end{array}$ & $\begin{array}{l}\text { Randomized clinical trial (pilot) } \\
\mathrm{N}=18\end{array}$ & $\begin{array}{l}\text { Development of a self-management support } \\
\text { protocol for oral care in the prevention of mucositis. }\end{array}$ & $\begin{array}{l}\text { Intervention/ } \\
\text { Treatment or Diagnosis/ } \\
\text { Diagnostic Test//l§ }\end{array}$ \\
\hline Treister et al. ${ }^{(45)}$ & $\begin{array}{l}\text { 2016/ } \\
\text { United States } \\
\text { of America }\end{array}$ & $\begin{array}{l}\text { Quasi-experimental study } \\
\mathrm{N}=13\end{array}$ & $\begin{array}{l}\text { Viability of low-level light therapy or combined } \\
\text { photobiomodulation therapy for the prevention of } \\
\text { mucositis. }\end{array}$ & $\begin{array}{l}\text { Intervention/ } \\
\text { treatment or diagnosis/ } \\
\text { diagnostic test/III } \dagger\end{array}$ \\
\hline
\end{tabular}

Note: *evidence from a single cohort or case-control study; tevidence from clinical trials without randomization; ‡evidence from a single qualitative or descriptive study; §evidence from randomized clinical trials. 
Chart 4 - Characterization of primary studies in the self-management category of care $(n=5)$, according to author(s), year of publication and country, type of study, intervention/focus, clinical issue and level of evidence, Curitiba, Paraná, Brazil

\begin{tabular}{|c|c|c|c|c|}
\hline Author(s) & Year/country & Type of study & Intervention/focus & $\begin{array}{l}\text { Clinical question/ } \\
\text { level of evidence }\end{array}$ \\
\hline Barban et al. ${ }^{(46)}$ & $\begin{array}{l}\text { 2014/ } \\
\text { Brazil }\end{array}$ & $\begin{array}{l}\text { Retrospective, quantitative, } \\
\text { descriptive, cross-sectional } \\
\text { study } \mathrm{N}=110\end{array}$ & $\begin{array}{l}\text { Results of hospital discharge as a viable alternative } \\
\text { in treatment. }\end{array}$ & $\begin{array}{l}\text { Prognosis/ } \\
\text { prediction or etiology/IV* }\end{array}$ \\
\hline Best et al. ${ }^{(47)}$ & $\begin{array}{c}2016 / \\
\text { United States } \\
\text { of America }\end{array}$ & $\begin{array}{l}\text { Quasi-experimental study, } \\
\text { time series model } \\
N=100\end{array}$ & $\begin{array}{l}\text { Creation and implementation of standardized } \\
\text { process of compliance with activities of daily living. }\end{array}$ & $\begin{array}{l}\text { Intervention/ } \\
\text { treatment or diagnosis/ } \\
\text { diagnostic test/IIt }\end{array}$ \\
\hline Castro et al. ${ }^{(48)}$ & $\begin{array}{l}2012 / \\
\text { Brazil }\end{array}$ & $\begin{array}{l}\text { Qualitative study } \\
\mathrm{n}=7\end{array}$ & $\begin{array}{l}\text { The influence of orientations provided at discharge } \\
\text { on self-care after transplantation. }\end{array}$ & Meaning/ll‡ \\
\hline Schmidt et al. ${ }^{(49)}$ & $\begin{array}{l}\text { 2017/ } \\
\text { Germany }\end{array}$ & $\begin{array}{l}\text { Prospective intervention study } \\
\text { (non-randomized) } \\
\mathrm{N}=79\end{array}$ & $\begin{array}{l}\text { Intervention to improve patients'self-management } \\
\text { skills regarding activation and relaxation, prevention } \\
\text { of oral mucositis and malnutrition. }\end{array}$ & $\begin{array}{l}\text { Intervention/ } \\
\text { treatment or diagnosis/ } \\
\text { diagnostic test/lli }\end{array}$ \\
\hline Thomson et al. ${ }^{(50)}$ & $\begin{array}{l}\text { 2015/ } \\
\text { United States } \\
\text { of America }\end{array}$ & $\begin{array}{l}\text { Quasi-experimental study } \\
\mathrm{N}=22\end{array}$ & $\begin{array}{l}\text { Minimization of the variability of teaching content } \\
\text { and improvement of nurses' competence. }\end{array}$ & $\begin{array}{l}\text { Intervention/ } \\
\text { treatment or diagnosis/ } \\
\text { diagnostic test/III }+\end{array}$ \\
\hline
\end{tabular}

Note: *evidence from a single qualitative or descriptive study; tevidence from clinical trials without randomization; ¥evidence from a single qualitative study.

\section{DISCUSSION}

A considerable part of the primary studies included in the review was carried out in the USA $(n=17)$, a developed country, especially in terms of transplantation, which stimulates the production of knowledge in the most diverse areas and professions. Moreover, the use of English language in publications, including Brazilian ones, highlights a growing trend in the dissemination of this language.

In the first category, multiple nursing care, the authors of the primary studies investigated different care, the activities of nurses and or nursing staff, considering the different stages and contexts of post-HSCT ${ }^{(11-29)}$.

The application of a patients' decline assessment scale included assessment of the level of consciousness, capillary filling, heart rate, respiratory pattern and frequency, oxygen saturation, oxygen support and elevation of the head of the bed. Monitoring vital signs has been discussed in seven other studies ${ }^{(11,14,20,22,24-26)}$, pointing out the relevance of this care in the assessment of the clinical condition of patients undergoing transplantation. In this perspective, the authors of other studies ${ }^{(17,20,25-26,28)}$ also investigated clinical assessment, medication administration and/or the use of Systematization of Nursing Care (SNC).

In the context of GVHD, the main precautions were: infection control; fall prevention; monitoring of fatigue; food and water intake; anxiety reduction; relaxation therapy; assistance in self-care; skin care; diarrhea control ${ }^{(12)}$. The last two cares have been investigated in more studies ${ }^{(15,26)}$. GVHD is one of the most serious complications in allogeneic post-transplantation, which can compromise quality of life, which is why it requires planned interventions for its assessment and control.

In the research on home care in the post-HSCT, blood collection, supportive care and analysis of clinical and laboratory data, through the visit and home care, were the issues investigated with prominence ${ }^{(13)}$. Home visit was also a strategy defined for care in yet another study ${ }^{(16)}$. Blood collection was discussed in four other studies ${ }^{(18,20,25-26)}$, two of which were related to the best practices for the collection of CVC and peripheral blood cultures.

In two studies ${ }^{(15-16)}$, the post-discharge guidelines provided for patients were: oral hygiene; be careful with venous access; signs and symptoms to report; prevention of dehydration; medication review; precautionary information; diet to prevent infections, GVHD, community and hospital resources; fluid support; displacement of the family's role; adjustment after hospitalization; offering support; coping with life after treatment. In the meantime, it is clear that the care demands are not just physical, and that the social and psychological impact caused by HSCT was considered.

Practices to improve medication adherence, such as assessing adherence, training patients to take medication, and using strategies, such as educational materials, were the issue investigated in a study ${ }^{(19)}$.

In two studies ${ }^{(20,24)}$, nurses' activities on admission to HSCT were studied, highlighting: body hydration; verification of oxygen saturation, weight and capillary glycemia; hand hygiene; control of water balance and drug infusion; maintenance of CVC; preparation of intravenous substances in laminar flow; care with blood transfusion, parenteral nutrition and chemotherapy; stem cell infusion; oxygen therapy management; availability to listen. Hand hygiene was scored in just one more study ${ }^{(25)}$.

Alternative interventions, performed by nurses, were discussed in a single study ${ }^{(21)}$, namely: pain drainage; Chakra connection; magnetic clearing and clearing the mind; nutritional and hydration support; pain control; protective insulation; physical rehabilitation therapy; recreational therapies; mental health; pastoral counseling. Pain management and use of analgesia were investigated in another study ${ }^{(28)}$.

In four studies ${ }^{(21,23,25-26)}$, the authors discussed the role of nurses in the indication and implementation of enteral nutrition and/or parenteral nutrition, with parents of children undergoing HSCT in the event of decreased oral intake or loss of weight.

In research ${ }^{(25)}$ included in the review, the main care proposed was: investigation of the dietary pattern; assessment of physiological disposal systems and laboratory tests; anamnesis and physical examination; isolation of colonized patients or those with 
respiratory infections; guidance on care for the catheter at home; hand washing; hygiene and maintenance of the environment; early administration of antibiotics and antivirals.

In a prospective cohort study ${ }^{(26)}$, the following nursing care was addressed: drain management; mobilization and positioning; use of vasoactive medication; intravenous fluid replacement; cardiorespiratory resuscitation; treatment of metabolic acidosis or alkalosis; oral cryotherapy; infection risk and prevention guidelines.

In a survey ${ }^{(27)}$, breaking the bad news was the central focus, aiming to minimize psychological impacts and protect patients from disturbing information by providing partial information and omitting details.

In the second category, first-line care, in the subcategory preventive infection care, the investigated actions had as main focus the prevention of infections $s^{(2,5,30-35)}$. The use of masks by patients and standard precautions by the team and care in the collection of feces and blood cultures were addressed ${ }^{(30)}$, the latter being pointed out in two more studies ${ }^{(31,34)}$. The inclusion of these studies in this category, and not in the first, was based on the focus of nursing care, i.e., preventive, and not only as an action in the management of patients already with fever and possible infection installed.

In three studies ${ }^{(5,31,33)}$, the results pointed out the importance of the use of a catheter bundle by nursing, including measures such as: hand washing; disinfection of hands with alcohol; disinfection of the catheter ends; use of transparent film; use of personal protective equipment; care practices with intravenous tubing; maintaining sterility of the distal extremity.

Other precautions to prevent infection were: use of a High Efficiency Particulate Arrestance (HEPA) filter; preparation of medication in laminar flow; screening of visitors; guidelines regarding the use of masks and hand washing, care for the environment; isolation; nutritional guidelines; maintenance of venous access; oral care; CVC protection for the bath; avoid contact with children with contagious diseases or who have received vaccines; avoiding crowds, places with works; caregiver and patient education for post-discharge care ${ }^{(5)}$. The use of a HEPA filter was pointed out in another study ${ }^{(35)}$.

In other studies ${ }^{(2,33-34)}$, the care discussed was checking vital signs; early initiation of antibiotic therapy; integrated communication with the team involved in care; patient hygiene; preparation of chemotherapeutic agents and parenteral nutrition; dialogue with the multidisciplinary team about a possible case of sepsis; verification of capillary perfusion; collection and assessment of laboratory tests.

In a study ${ }^{(32)}$, nursing care in assessing the degree of GVHD and the amount of diarrhea was investigated, since this condition is a risk factor for infection. Assessing blood count and digestive symptoms, maintaining protective isolation in neutropenia, performing rigorous aseptic procedures, avoiding cross-infection and restricting visitors and comforting patients and their families.

In the subcategory care with central venous catheter, studies focused on catheter care ${ }^{(36-41)}$, the use of this device being fundamental for HSCT to happen. The insertion dressing was recommended using aseptic technique, with $0.5 \%$ alcoholic chlorhexidine and gauze, occluded with adhesive tape and maintained for 24 hours after implantation of the catheter and/ or in the presence of exudate or moisture, or using transparent film by up to seven days if insertion without secretion, in addition to daily assessment of insertion. Insertion time assessment, the history of allergies to adhesive tape and skin conditions and the replacement of alcoholic chlorhexidine with $0.9 \%$ saline solution, in case of skin injury, were also investigated.

Other precautions discussed were: proper maintenance of the device, with disinfection of the hub with alcohol or chlorhexidine before accessing the system; washing with saline and blocking with heparin; daily replacement of the CVC cover; protection for the bath and the extremities with gauze and micropore; clearing the pathways using anticoagulants; preparation and assessment of blood culture results reports; diagnoses of different types of infection; guidance of care for patients and family members; early removal of the device ${ }^{(38-41)}$.

In the care with mucositis subcategory, primary studies on prevention and treatment of this problem were grouped ${ }^{(42-45)}$. The care provided was daily assessment of the severity of mucositis, application of a graduation scale, education in oral hygiene, cryotherapy guidance and assessment of adherence to medication to prevent/control oral mucositis. General guidelines on the use of rinse aid and medications were also present, such as rinsing with chlorhexidine gluconate (when there are no signs of mucositis), normal saline, magic mouthwash, use of Caphosol ${ }^{\circ}$ mouthwash and Cepastat ${ }^{\circ}$ lozenges, palifermin, mouthwash with chamomile, administration of opioids and total parenteral nutrition. Daily combined photobiomodulation therapy (PBT) was carried out in a quasi-experimental study.

In the third category, self-management of care, the authors of the grouped primary studies investigated care with a focus on preparation and assessment for self-care ${ }^{(46-50)}$. The recommendations discussed were: information to patients and their caregivers about the adverse effects of treatment; assessment of fatigue, anxiety, insomnia, lack of appetite, mucositis, nausea, and vomiting; advice on how to identify possible changes and the need to seek professional assistance; baths with chlorhexidine and change of clothes daily; oral hygiene care two to three times a day. Other precautions were exercise supervision, provision of information and motivational support, follow-up and monitoring of food intake and counseling of patients on frequent nutritional problems $s^{(46-47,49)}$.

In another study, the following guidelines for self-care were studied: avoid agglomerations or people with an infectious chart; select and handle food properly; drink mineral water; washing the fruit; wear a mask; having a companion to clean the house; moisturize the skin with body moisturizer; use sunscreen; contact team if fever; not having contact with domestic animals; stay in a hygienic environment ${ }^{(48)}$.

In another study ${ }^{(50)}$, the actions discussed comprised: coordination of outpatient treatment and hospitalization; scheduling posttransplant medical appointments; observation and assessment of teaching competence; participation in educational sessions; discharge planning; planning assessment; early identification of potential barriers, educational needs, family conferences and follow-up by phone calls; effort to decrease the burden of care for patients and caregivers; help to relieve anxiety for patients and caregivers at the time of transition from various levels of care and locations, providing education. 
It is noteworthy that the studies discussed focused on the prevention, identification and/or treatment of clinical conditions expected post-HSCT, monitoring these patients is extremely important. In addition to addressing actions aimed at alleviating physical suffering, psychological and social aspects were also discussed.

As for the provision of care by nurses or the nursing team, it should be noted that the composition of the latter is different, comparing Brazil with other countries. In the latter, there is only the nurse (professional with a higher education) responsible for nursing activities, which may have contributed to the number of citations of nurses. In an analysis of the set of included primary studies, it is clear the relevance of nurses' role in the care of post-HSCT patients, especially in daily technical care in the hospital environment and in the provision of orientations directed at home care.

\section{Study limitations}

The selection of primary studies for the composition of this review is considered a limitation, since gray literature was not part, as well as the restriction of languages and data analysis in a descriptive way, associating studies from different approaches.

\section{Contributions to nursing, health, and public policies}

The nursing care provided to patients in post-HSCT is varied, being of different complexity. In general, the following stand out: constant clinical assessment; infection control; collection and monitoring of laboratory tests; hydration; hydric balance; preparation and administration of medications, including analgesia; care with CVC; guidelines for self-care; social isolation; psychological support.

The evidence identified enables nursing professionals in HSCT to know the diversity of necessary and performed care at national and international level. Thus, the conducted review provides elements for the performance of these professionals, as well as points out gaps that lack studies that corroborate their development and the elaboration of public policies aimed at the needs of this clientele.

\section{CONCLUSIONS}

In an analysis of the knowledge synthesis generated in this review, it can be inferred that nursing care focuses on the physical aspects, especially, but also patients' psychological and social aspects. Evidence has shown that nursing care in HSCT: encompasses a multiplicity of actions by the nursing team; it is critical, therefore, many cares, if not performed, put patients lives at risk; it happens in hospital and home contexts; involves technical actions and guidance in health.

It also points out the diversity in practices, which compromises safe care. For this, the synthesis of knowledge produced by this review provides instrumental to HSCT nurses about the actions that they perform and that are necessary, as well as providing subsidies for the construction of standardized care plans, such as care protocols. It is noteworthy that most studies are of the nonexperimental type, indicating the need to conduct intervention studies, which can contribute with strong evidence on the issue, especially in Brazil.

\section{REFERENCES}

1. Associação Brasileira de Transplante de Órgãos (ABTO). Registro Brasileiro de Transplantes: estatística de transplantes [Internet]. Brasil: ABTO; 2019 [cited 2020 Jan 22]. Available from: http://www.abto.org.br/abtov03/Upload/file/RBT/2019/RBT-2019-jan-set-leitura.pdf

2. Daniels $\mathrm{P}$, Pate A, Flesch L, Teusink-Cross A, Matani H, Geiger A, et al. Improving time to antibiotic administration for bone marrow transplant patients with first fever. Pediatrics. 2018;141(1). https://doi.org/10.1542/peds.2017-1549

3. Rodrigues JAP, Lacerda MR, Gomes IM, Paes MR, Ribeiro RP, Bonfim CMS. Clinical profile of children undergoing hematopoietic stem cell transplantation. Cogitare Enferm. 2019;24. https://doi.org/10.5380/ce.v24i0.55967

4. Young LK, Mansfield B, Mandoza J. Nursing care of adult hematopoietic stem cell transplant patients and families in the intensive care unit: an evidence-based review. Crit Care Nurs Clin North Am. 2017;29(3):341-52. https://doi.org/10.1016/j.cnc.2017.04.009

5. Bevans M, Tierney DK, Bruch C, Burgunder M, Castro K, Ford R, et al. Hematopoietic stem cell transplantation nursing: a practice variation study. Oncol Nurs Forum. 2009;36(6):317-25. https://doi.org/10.1188/09.0NF.E317-E325

6. Gomes IM, Lacerda MR, Hermann AP, Rodrigues JAP, Zatoni DCP, Tonin L. Care performed by family caregivers of children submitted to hematopoietic stem cell transplantation. Rev Latino-Am. Enfermagem. 2019;27:e3120. https://doi.org/10.1590/1518-8345.2298-3120

7. Whittemore R, Knafl K. The integrative review: updated methodology. J Adv Nurs. 2005;52(5):546-53. https://doi. org/10.1111/j.1365-2648.2005.03621.x

8. Fineout-Overholt E, Stillwell SB. Asking compelling, clinical questions. In: Melnyk B M, Fineout-Overholt E. Evidence-based practice in nursing and healthcare: a guide to best practice. Philadelphia: Wolters Kluwer; 2018. p. 33-54.

9. Ursi ES, Galvão CM. Prevenção de lesões de pele no perioperatório: revisão integrativa da literatura. Rev Latino-Am Enfermagem. 2006;14(1):124-31. https://doi.org/10.1590/S0104-11692006000100017

10. Polit DF, Beck CT, Hungler BP. Fundamentos de pesquisa em enfermagem métodos, avaliação e utilização. 5th ed. Porto Alegre: Artmed; 2011.

11. Agulnik A, Forbes PW, Stenquist N, Rodriguez-Galindo C, Kleinman M. Validation of a Pediatric Early Warning Score in hospitalized pediatric oncology and hematopoietic stem cell transplant patients. Pediatr Crit Care Med. 2016;17(4):146-53. https://doi.org/10.1097/ PCC.0000000000000662 
12. Araujo DD, Rodrigues AB, Oliveira PP, Silva LS, Vecchia BP, Silveira EAA. Nursing diagnoses and interventions for patients with graft-versushost disease submitted to hematopoietic stem cell transplantation. Cogitare Enferm [Internet]. 2015 [cited 2019 Sept 7];20(2):305-13. Available from: http://docs.bvsalud.org/biblioref/2016/08/1262/40340-157233-1-pb.pdf

13. Bergkvist K, Larsen J, Johansson UB, Mattsson J, Svahn BM. Hospital care or home care after allogeneic hematopoietic stem cell transplantation - patients' experiences of care and support. Eur J Oncol Nurs. 2013;17(4):389-95. https://doi.org/10.1016/j.ejon.2012.12.004

14. Cater DT, Tori AJ, Moser EAS, Rowan CM. Modification and assessment of the bedside pediatric early warning score in the pediatric allogeneic hematopoietic cell transplant population. Pediatr Crit Care Med. 2018;19(5):483-8. https://doi.org/10.1097/ PCC.0000000000001521

15. Cooke L, Grant M, Gemmill R. Discharge needs of allogeneic transplantation recipientes. Clin J Oncol Nurs. 2012;16(4):142-9. https://doi. org/10.1188/12.CJON.E142-E149

16. Cooke LD, Gemmill R, Grant ML. Creating a palliative educational session for hematopoietic stem cell transplantation recipients at relapse. Clin J Oncol Nurs. 2011;15(4):411-7. https://doi.org/10.1188/11.CJON.411-417

17. Ferreira M, Nascimento LC, Braga FTMM, Silva-Rodrigues FM. Nurses' competences in the critical care of children undergoing hematopoietic stem cell transplantation. Rev Eletrôn Enferm. 2017;19. https://doi.org/10.5216/ree.v19.43604

18. Gori E, Callea E, Alberani F, Orlando L. Microbial monitoring and methods of sample collection: a GITMO survey. Ecancer Med Sci. $2014 ; 8: 421$. https://doi.org/10.3332/ecancer.2014.421

19. Kirsch M, Berben L, Johansson E, Calza S, Eeltink C, Stringer J, et al. Nurses' practice patterns in relation to adherence-enhancing interventions in stem cell transplant care: a survey from the Nurses Group of the European Group for Blood and Marrow Transplantation. Eur J Cancer Care (Engl). 2014;23(5):607-15. https://doi.org/10.1111/ecc.12172

20. Lima K, Bernardino E. Nursing care in a hematopoietic stem cells transplantation unit. Texto \& Contexto Enferm. 2014;23(4):845-53. https:// doi.org/10.1590/0104-07072014000440013

21. Lu DF, Hart LK, Lutgendorf SK, Oh H, Silverman M. Effects of healing touch and relaxation therapy on adult patients undergoing hematopoietic stem cell transplant: a feasibility pilot study. Cancer Nurs. 2016;39(3):1-11. https://doi.org/10.1097/NCC.0000000000000272

22. Meseguer-Liza C, Caravaca-Hernández A, Solano-Antolinos J, López-Martínez S, Meseguer-Sánches, Sánches-Martínez C, et al. Prospective study of tympanic temperature in patients undergoing haemopoietic transplant. Enferm Clin. 2010;20(3):147-52. https://doi.org/10.1016/j. enfcli.2010.01.008

23. Montgomery K, Belongia M, Schulta C, Mulberry MH, Nugent ML, Simpson PM. Health Care Providers' Perceptions of Nutrition Support in Pediatric Oncology and Hematopoietic Stem Cell Transplant Patients. J Pediatr Oncol Nurs. 2016;33(4):265-72. https://doi. org/10.1177/1043454215616604

24. Rodrigues C, Pinto C, Pereira F. A pessoa submetida a transplante de medula óssea: estudo exploratório sobre os padrões de documentação de cuidados de enfermagem. Onco News [Internet]. 2013 [cited 2019 Sept 8];22:17-22. Available from: https://www.aeop.pt/ficheiros/39438 2b2324b245084cfa33d4dd0f6f78.pdf

25. Rodrigues JAP, Lacerda MR, Gomes IM, Paes MR, Ribeiro RP, Mercês NNA. Research contributions for the nursing care in pediatric transplantation of hematopoietic stem cells. Rev Pesqui Cuid Fundam. 2018;10(4):964-70. https://doi.org/10.9789/2175-5361.2018 v10i4.964-970

26. Silva JB, Póvoa VCO, Lima MHM, Oliveira HC, Padilha KG, Secoli SR. Nursing workload in hematopoietic stem cell transplantation: a cohort study. Rev Esc Enferm USP. 2015;49(Esp):92-8. https://doi.org/10.1590/S0080-623420150000700014

27. Valizadeh L, Zamanzadeh V, Sayadi L, Taleghani F, Howard AF, Jeddian A. Truth-telling and hematopoietic stem cell transplantation: Iranian nurses' experiences. Nurs Ethics. 2014;21(5):518-29. https://doi.org/10.1177/0969733013511359

28. Vasquenza K, Ruble K, Chen A, Billett C, Kozlowski L, Atwater S, et al. Pain Management for Children during Bone Marrow and Stem Cell Transplantation. Pain Manag Nurs. 2015;16(3):156-62. https://doi.org/10.1016/j.pmn.2014.05.005

29. Zamanzadeh V, Valizadeh L, Sayadi L, Taleghani F, Howard F, Jeddian A. Emotional labour of caring for hematopoietic stem cell transplantation patients: Iranian nurses' experiences. Asian Nurs Res. 2013;7(2):91-7. https://doi.org/10.1016/j.anr.2013.04.004

30. Balian C, Garcia M, Ward J. A retrospective analysis of bloodstream infections in pediatric allogeneic stem cell transplant recipients: the role of central venous catheters and mucosal barrier injury. J Pediatr Oncol Nurs. 2018;35(3):210-7. https://doi.org/10.1177/1043454218762706

31. Barrell C, Covington L, Bathia M, Robison J, Patel S, Jacobson JS, et al. Preventive strategies for central line-associated bloodstream infections in pediatric hematopoietic stem cell transplant recipients. Am J Infect Control. 2012;40(5):434-9. https://doi.org/10.1016/j.ajic.2011.06.002

32. Hou C, Xu L, Chen H, Liu N, Jiang M, Wang G, et al. Intestinal aGVHD and infection after hematopoietic stem cell transplantation. Med Sci Monit. 2013;19:802-6. https://doi.org/10.12659/MSM.889408

33. Livadiotti S, Milano GM, Serra A, Folgori L, Jenkner A, Castagnola E, et al. A survey on hematology-oncology pediatric AIEOP centers: prophylaxis, empirical therapy and nursing prevention procedures of infectious complications. Haematol. 2012;97(1):147-50. https://doi. org/10.3324/haematol.2011.048918

34. Shelton BK, Stanik-Hutt J, Kane J, Jones RJ. Implementing the surviving sepsis campaign in an ambulatory clinic for patients with hematologic malignancies. Clin J Oncol Nurs. 2016;20(3):281-8. https://doi.org/10.1188/16.CJON.281-288 
35. Vokurka S, Bystrická E, Svoboda T, Skoda G, Sever M, Mazur E, et al. The availability of HEPA-filtered rooms and the incidence of pneumonia in patients after haematopoietic stem cell transplantation (HSCT): results from a prospective, multicentre, eastern European study. J Clin Nurs. 2014;23(11-12):1648-52. https://doi.org/10.1111/jocn.12286

36. Andrade AM, Borges KS, Lima HO. Cost analysis and evaluation of covers for a central venous catheter insertion site in BMT. REME Rev Min Enferm. 2011;15(2):233-41. https://doi.org/S1415-27622011000200011

37. Silveira RCCP, Braga FTMM, Garbin LM, Galvão CM. The use of polyurethane transparent film in indwelling central venous catheter. Rev Latino-Am Enfermagem. 2010;18(6):1212-20. https://doi.org/10.1590/S0104-11692010000600023

38. Flynn JM, Keogh SJ, Gavin NC. Sterile v aseptic non-touch technique for needle-less connector care on central venous access devices in a bone marrow transplant population: a comparative study. Eur J Oncol Nurs. 2015;19(6):694-700. https://doi.org/10.1016/j.ejon.2015.05.003

39. Keeler M. Central line practice in Canadian blood and marrow transplant. Can Oncol Nurs J. 2014;24(2):67-71. https://doi. org/10.5737/1181912×2426771

40. Carvalho ALG, Rosa LM. Dressing of central venous catheter of short permanence: nursing care. J Nurs UFPE. 2014;8(7):1972-8. https://doi. org/ 10.5205/5941

41. Yilmaz MC, Aksoylar S, Erdogan D, Demirag B. Complications of central venous catheters in children undergoing hematopoietic stem cell transplantation in Turkey. J Pediatr Oncol Nurs. 2012;29(4):199-205. https://doi.org/10.1177/1043454212451366

42. Bhatt V, Vendrell N, Nau K, Crumb D, Roy V. Implementation of a standardized protocol for prevention and management of oral mucositis in patients undergoing hematopoietic cell transplantation. J Oncol Pharm Pract. 2010;16(3):195-204. https://doi. org/10.1177/1078155209348721

43. Braga FT, Santos AC, Bueno PC, Silveira RC, Santos CB, Bastos JK, et al. Use of chamomilla recutita in the prevention and treatment of oral mucositis in patients undergoing hematopoietic stem cell transplantation: a randomized, controlled, phase II clinical trial. Cancer Nurs. 2015;38(4):322-9. https://doi.org/10.1097/NCC.0000000000000194

44. Leppla L, De Geest S, Fierz K, Deschler-Baier B, Koller A. An oral care self-management support protocol (OrCaSS) to reduce oral mucositis in hospitalized patients with acute myeloid leukemia and allogeneic hematopoietic stem cell transplantation: a randomized controlled pilot study. Support Care Cancer. 2016;24(2):773-82. https://doi.org/10.1007/s00520-015-2843-1

45. Treister NS, London WB, Guo D, Malsch M, Verrill K, Brewer J, et al. A feasibility study evaluating extraoral photobiomodulation therapy for prevention of mucositis in pediatric hematopoietic cell transplantation. Photomed Laser Surg. 2016;34(4):178-84. https://doi.org/10.1089/ pho.2015.4021

46. Barban A, Coracin FL, Musqueira PT, Barban A, Ruiz LP, Ruiz MA, et al. Analysis of the feasibility of early hospital discharge after autologous hematopoietic stem cell transplantation and the implications to nursing care. Rev Bras Hematol Hemoter. 2014;36(4):264-8. https://doi. org/10.1016/j.bjhh.2014.05.003

47. Best D, Osterkamp E, Demmel K, Kiniyalocts S, Mock S, Mulligan K, et al. Increasing activities of daily living is as easy as 1-2-3. J Pediatr Oncol Nurs. 2016;33(5):345-52. https://doi.org/10.1177/1043454215616607

48. Castro EAB, Andrade AM, Santos KB, Soares TC, Esterci LT. Self-care after autologous bone marrow transplantation within the nursing care process. Rev Rene [Internet]. 2012 [cited 2019 Sept 7];13(5):1152-62. Available from: http://periodicos.ufc.br/rene/article/view/4126/3213

49. Schmidt H, Boese S, Bauer A, Landenberger M, Lau A, Stoll O, et al. Interdisciplinary care programme to improve self-management for cancer patients undergoing stem cell transplantation: a prospective non-randomised intervention study. Eur J Cancer Care (Engl). 2017;26(4). https://doi.org/10.1111/ecc.12458

50. Thomson B, Gorospe G, Cooke L, Giesie P, Johnson S. Transitions of care: a hematopoietic stem cell transplantation nursing education project across the trajectory. Clin J Oncol Nurs. 2015;19(4):74-9. https://doi.org/10.1188/15.CJON.E74-E79 